\title{
Anomalous electron mobility modeling in Hall thrusters
}

\author{
Justin W. Koo* and Iain D. Boyd ${ }^{\dagger}$ \\ University of Michigan, Ann Arbor, MI, 48104, USA
}

\begin{abstract}
Accurate modeling of the anomalous electron mobility is crucial for successful simulation of Hall thrusters. Existing computational models for the anomalous electron mobility are used to simulate the UM/AFRL P5 Hall thruster in a 2D axisymmetric hybrid PIC-MCC code. Comparison to experimental results indicates that while these computational models can be tuned to reproduce the correct thrust or discharge current, it is very difficult to match all integrated performance parameters (thrust, power, discharge current, etc.) simultaneously. Furthermore, multiple configurations of these computational models can produce reasonable integrated performance parameters. Mean centerline potential profiles from these simulations are presented to provide physical insight into the relationship between the electron mobility and the spatial variation of the plasma potential.
\end{abstract}

\section{Nomenclature}

$\mu_{e} \quad$ Electron mobility perpendicular to magnetic field

$e \quad$ Electron charge

$m \quad$ Electron mass

$\nu_{m} \quad$ Electron momentum collision frequency

$\nu_{\text {neut }}$ Electron-neutral collision frequency

$\omega_{B, e}$ Electron cyclotron frequency

$n_{a} \quad$ Neutral density

$n_{e} \quad$ Plasma density

\section{Motivation}

Among the many modeling choices necessary in the computational simulation of Hall thrusters, the modeling of one particular physical phenomenon, the electron mobility, a measure of the response of the electrons to an applied electric field, stands out as a particularly difficult task. This is due to the relative lack of experimental measurements of the electron mobility and an incomplete understanding of the particular physics associated with this phenomenon.

A principle characteristic of Hall thrusters is the relatively high electron current which moves from the cathode towards the anode across the largely radial magnetic field in the acceleration channel. (The cathode provides electrons for both the discharge current and to neutralize the plume as shown in Fig. 1). To generate this high electron current observed experimentally, it is necessary in computer simulations to consider an electron mobility perpendicular to the magnetic field, $\mu_{e}$, that is larger than predicted by classical theory. (For the remainder of this paper, the electron mobility perpendicular to the magnetic field is simply referred to as the electron mobility.)

Additional electron transport mechanisms have been proposed to reflect this increased electron mobility, but no single mechanism has proved completely successful in modeling the observed behavior of the electron mobility. Since the enhanced electron mobility is so fundamental to thruster operation, it is difficult to isolate its effect experimentally; nevertheless, correctly modeling this additional electron transport mechanism, also

*Research Fellow, Aerospace Engineering Department, and AIAA Student Member.

${ }^{\dagger}$ Professor, Aerospace Engineering Department, and AIAA Fellow. 


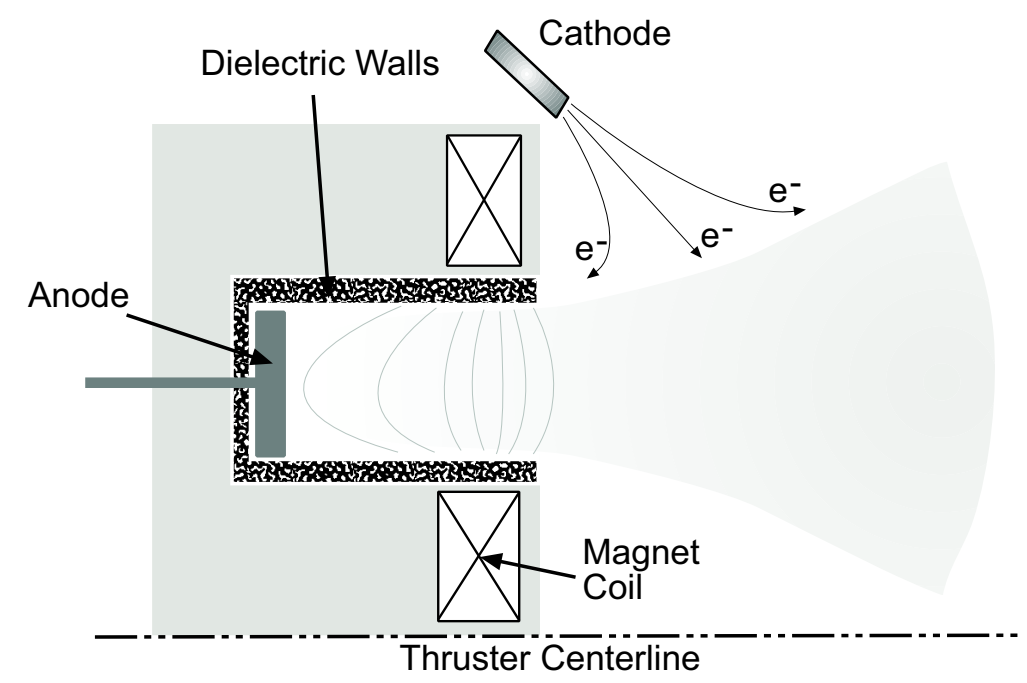

Figure 1. Dielectric wall Hall thruster schematic - Acceleration channel refers to region bounded by dielectric walls and near-field refers to region downstream of thruster face

known as the anomalous electron mobility, is absolutely critical to the successful numerical simulation of Hall thrusters.

This paper details the particular thruster operating condition used in this work. A brief overview of existing anomalous electron mobility models is then presented. Finally, simulation results using a combination of anomalous electron mobility models are presented and discussed.

\section{Hall Thruster Model}

All the computational simulations presented in this paper model a single operating condition of the UM/AFRL P5 Hall thruster running on xenon propellant. Full details of this operating condition are provided in Table 1. The computational simulations presented in this paper are produced with a 2-D axisymmetric hybrid PIC-MCC Hall thruster code developed by Koo. ${ }^{1,2}$ The physics governing this code are similar to those simulated in codes by $\mathrm{Fife}^{3}$ and Hagelaar et al. ${ }^{4}$ In particular, neutrals and ions are modeled with a Particle-In-Cell formulation while the electron fluid is governed by a 1D energy equation assuming isothermal behavior along magnetic field lines. The electrostatic potential is calculated from the thermalized potential via a quasi $1 \mathrm{D}$ current conservation approach relying on the magnetic field geometry for spatial discretization. Finally, a Monte Carlo Collision algorithm is used to evaluate ionization behavior.

\section{Electron Mobility Modeling}

The classical formulation for electron mobility perpendicular to magnetic field lines is:

$$
\mu_{e}=\frac{e}{m \nu_{m}} \frac{1}{1+\frac{\omega_{B, e}^{2}}{\nu_{m}^{2}}}
$$

where the electron cyclotron frequency is written as:

$$
\omega_{B, e}=\frac{e B}{m}
$$

and the electron momentum collision frequency, $\nu_{m}$, considered to dominated by the electron-neutral collision frequency, is evaluated as:

$$
\nu_{m} \approx \nu_{\text {neut }}=n_{a} \times 2.5 \cdot 10^{-13}
$$


Table 1. UM/AFRL P5 $3.0 \mathrm{~kW}$ Xe Performance ${ }^{a}$

\begin{tabular}{lcc}
\hline & Experimental & Uncertainty \\
\hline Discharge Voltage (V) & 300 & \\
Discharge Current (A) & 10 & \\
Thrust (mN) & 180 & $+1.2 /-8$ \\
Anode mass flow rate xenon (mg/s) & $10.248^{b}$ & \\
$I_{s p}(\mathrm{~s})$ & $1744^{c}$ & $+20 /-131^{d}$ \\
Efficiency (\%) & 51 & $+1 /-8.3$ \\
Background Pressure (torr) & $1.1 \cdot 10^{-5 e}$ & \\
\hline
\end{tabular}

${ }^{a}$ Cathode Mass Flow Rate $-0.586 \mathrm{mg} / \mathrm{s}$ xenon

$b_{2}$-D internal and near-field data taken at $10.736 \mathrm{mg} / \mathrm{s}$

${ }^{c}$ Actual $I_{s p}=1650 \mathrm{~s}$ presented in ${ }^{5}$ is based on anode plus cathode mass flow rates

${ }^{d}$ Uncertainty based on $I_{s p}=1650 \mathrm{~s}$

${ }^{e} 2$-D internal and near-field data taken at $3.2 \cdot 10^{-5}$ torr

A number of models for the anomalous electron mobility have been developed to account for the enhanced electron mobility observed in Hall thrusters. In this work, the various anomalous electron mobility models are assumed to act as additional collision mechanisms and are implemented through augmentation of the electron momentum transfer frequency. The primary computational models for the anomalous electron mobility are now described.

The wall-collision correction to the $\nu_{m}$ term, based on the idea that electrons collide with the sheath with a relative frequency based on their thermal energy and the width of the acceleration channel, has been developed by Boeuf et al. ${ }^{6}$ A simple form for this correction can be obtained by using an a priori averaged thermal energy to calculate a global wall-collision rate. For these computations, the modified form of the electron momentum transfer frequency (with wall-collisions), where $\alpha$ is an empirically chosen coefficient, is as follows:

$$
\nu_{m}=\nu_{n e u t}+\alpha \cdot 10^{7}
$$

The Bohm diffusion correction to the $\nu_{m}$ term is based on the idea that anomalous Bohm diffusion results in the additional electron mobility observed experimentally. For these computations, the modified form of the electron momentum transfer frequency, where $\alpha_{B}$ is an empirically chosen coefficient, is as follows:

$$
\nu_{m}=\nu_{\text {neut }}+\alpha_{B} \omega_{B, e}
$$

The wall-collision approach is based on the theory by Morozov et $\mathrm{al}^{7}$ that electron-wall collisions are the mechanism for enhancing electron transport inside the thruster. Accordingly, this theory is better suited to the acceleration channel (where there are walls) than to the near-field of the thruster (where there are no walls). The theory behind the mechanism for Bohm mobility, presented by Esipchuck et $a{ }^{8}{ }^{8}$ is based on transport from azimuthal drift waves in the plasma which can exist only in regions with decreasing gradients in the magnetic field. Consequently, the Bohm model for anomalous mobility is less applicable inside the thruster channel where the magnetic field criteria to support these azimuthal drift waves is not met.

To incorporate these ideas, mixed mobility models for Hall thrusters have been presented by Hagelaar et $\mathrm{al}^{4}$ with a wall-collision mobility model inside the thruster and a Bohm mobility model outside the thruster. The approach used in the present work to blend the two mobility regimes is slightly different than the approach presented by Hagelaar et al; ${ }^{4}$ however, the fundamental idea of using wall-collision mobility inside the thruster and Bohm mobility in the near-field is identical.

The use of a mixed mobility model, while better capturing the theoretical physics of the problem, introduces extra dimensions to the model parameter space. In this study, the boundary between the two models is fixed at the thruster exit and two parameters, $\alpha$ inside the channel and $\alpha_{B}$ outside the channel, are necessary to describe the model. 


\section{Performance of Anomalous Mobility Models}

The four different simulations presented in this section are all designed to simulate the same $3.0 \mathrm{~kW}$ xenon thruster configuration of the UM/AFRL P5 Hall thruster. Unless otherwise noted, the simulations presented employ reasonable model coefficients designed to best reflect the overall physical effects of the various mobility models. With any of the mobility models, individual performance metrics, such as thrust alone, can be matched more closely by using highly skewed model coefficients; however, such choices tend to force other performance parameters away from optimum.

The anomalous mobility models selected for this section are as follows: (1) Bohm diffusion mobility with $\alpha_{B}=0.005$ (Low Bohm), (2) Bohm diffusion mobility with $\alpha_{B}=0.015$ (High Bohm), (3) Mixed mobility: wall-collision mobility with $\alpha=0.15$ inside the acceleration channel and Bohm mobility with $\alpha_{B}=0.020$ outside the channel (Mixed Outer), and (4) Mixed mobility: wall-collision mobility with $\alpha=5.0$ inside the acceleration channel and Bohm mobility with $\alpha_{B}=0.008$ outside the channel (Mixed Inner). Mean centerline electron mobility data for all four models are presented in Fig. 2.

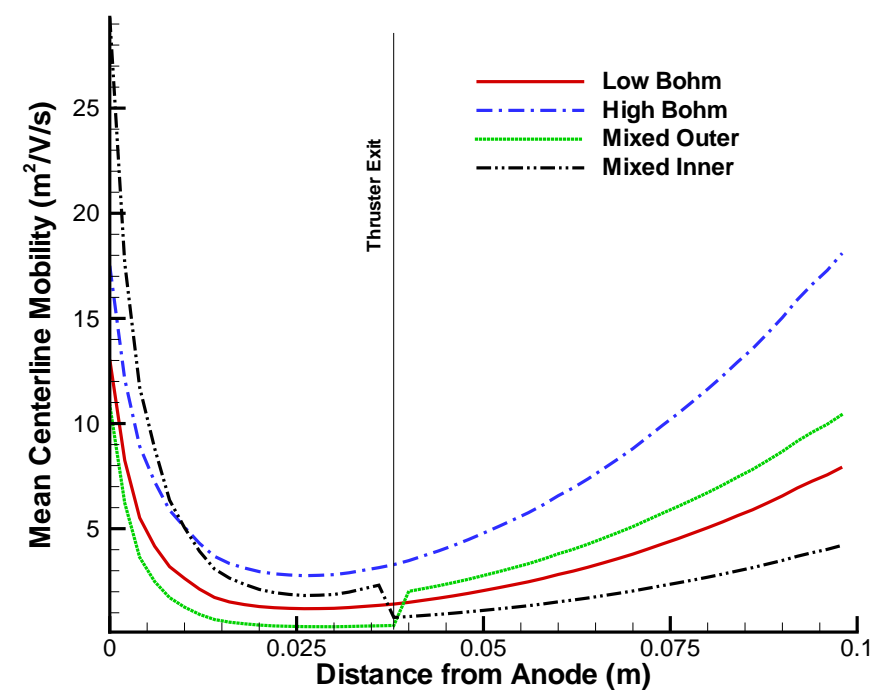

Figure 2. UM/AFRL P5 Mean Centerline Mobility

Table 2. UM/AFRL P5 Computational Mobility Model Performance Data

\begin{tabular}{lccccc}
\hline & Low Bohm & High Bohm & Mixed Outer & Mixed Inner & Measured \\
\hline Thrust (mN) & 154.5 & 182.7 & 169.4 & 173.9 & 180.0 \\
$I_{s p}(\mathrm{~s})$ & 1544 & 1824 & 1686 & 1728 & 1744 \\
Ion Current (A) & 11.07 & 11.58 & 9.90 & 10.92 & N/A \\
Electron Current (A) & 0.33 & 7.41 & 0.72 & 2.34 & N/A \\
Discharge Current (A) & 11.41 & 19.00 & 10.63 & 13.26 & 10.00 \\
Power (W) & 3137 & 5224 & 2922 & 3646 & 3000 \\
Thrust Efficiency (\%) & 37 & 31 & 48 & 40 & 51 \\
\hline
\end{tabular}

The integrated performance parameters produced by the four computational mobility models are shown in Table 2. The principal figure of merit for integrated performance data is the thrust. According to this criterion, the High Bohm and both Mixed mobility models perform quite successfully. The failing of the Low Bohm model is not surprising since it is optimized to the thruster power.

Another important listing in Table 2 is the electron current. As will become clear in this section, higher 
mobility coefficients generally result in higher electron currents. Since thrust performance is based largely on the ion current while the thruster power is based on the total current, simulations which produce comparable amounts of thrust can show wide variation in thruster power. In particular, while the High Bohm model provides almost exactly the experimentally observed level of thrust, it overpredicts the required power by over 70\%. On the other hand, the Mixed Outer model underpredicts the experimental thrust by only $6 \%$ while maintaining almost exactly the experimental power input. Since the thrust efficiency scales inversely with the discharge current, poor thruster efficiency results when the electron mobility (and, consequently, the electron current) is too large.

Internal and near-field time-averaged plasma potential data are presented in Fig. 4. Fig. 3(a) and Fig. 3(b) demonstrate the effect of increasing $\alpha_{B}$. (As desired, when $\alpha_{B} \cdot \omega_{c, e} \gg \nu_{n e u t}$, the correct scaling for $\mu$ in the limit of Bohm diffusion, $\mu \propto 1 / B$, is reached.) Since the $\alpha_{B}$ correction for the Low Bohm case is smaller than for the high Bohm case, the Low Bohm mobility case more closely resembles the flatter classical mobility. Conversely, greater positive curvature in the electron mobility profile is displayed by the High Bohm mobility. The different positive curvature in the mobility profiles can be clearly seen in the centerline mobility profiles for the two Bohm configurations in Fig. 2. For the Low Bohm case, the flatter mobility profile results in an almost linear potential gradient throughout the acceleration channel while for the High Bohm case, because the mobility drops more quickly in the channel and rises more quickly outside of the channel, the result is a much steeper potential gradient. Although the peak potential gradient is generally close to the minimum in the mobility curve, other factors, such as the electron current, electron temperature and pressure gradient, couple with the electron mobility to establish a self-consistent potential distribution. As a consequence, even though both Bohm mobility models have their minimum mobility in the same location, their highest electric fields do not coincide.

The results presented in Fig. 4(a) demonstrate the tendency of a high mobility in the near-field of the thruster (relative to inside the thruster) to force the acceleration zone into the acceleration channel. Figure 4(b) demonstrates the opposite behavior when the near-field of the thruster has a low mobility relative to the acceleration channel. By varying the strength of these coefficients, it is possible to control the magnitude of the potential drop inside the channel. It is not possible, within the framework of this particular implementation, to change the relative shape of the mobility curve beyond the single control parameter $(\alpha$ or $\alpha_{B}$ ) in a given region of the thruster. As a result, for the Mixed Inner mobility model, a small cusp in the mobility profile just upstream of the thruster exit is sufficient to induce a discontinuity in the gradient of the centerline potential just after the thruster exit.

Existing models for anomalous electron mobility offer a useful tool to rapidly evaluate possible thruster operating conditions. With proper parameter choices, some performance metrics can be reached by optimizing a single parameter (as in the Bohm model presented in this section); however, accurately simulating one integrated performance parameter in no way guarantees that all integrated performance parameters will be correct. Furthermore, fixed performance metrics can be achieved by multiple mobility models displaying vastly different internal and near-field plasma properties. Against this backdrop, more complicated, mixed mobility models have been developed which allow for more user control over the mobility profile while maintaining the theoretical underpinnings of these corrections. These mixed mobility models, when properly tuned, can produce the best combination of integrated performance parameters; however, establishing the correct parameters to achieve these results is still a very much ad hoc process.

\section{Conclusion}

Simulations of the discharge chamber and plume near-field of a Hall thruster based on Bohm diffusion and mixed anomalous mobility models revealed that these models can be tuned to provide fairly accurate thrust or discharge current performance; however, it is difficult to match all of the integrated performance parameters simultaneously. With the additional flexibility offered by the mixed mobility models, it is possible to better match the overall integrated performance parameters than with the single coefficient Bohm mobility model; however, since multiple coefficient settings provide fairly realistic solutions, it is not clear which mobility

profiles are physically correct and which resulting mobility profiles are actually correct only in the context of the reduced physics of the simulations. The success of future computational simulation of Hall thrusters will be strongly dependent on the availability of internal experimental data and proper analysis geared towards better understanding of the physics and applicability of anomalous electron mobility models. 

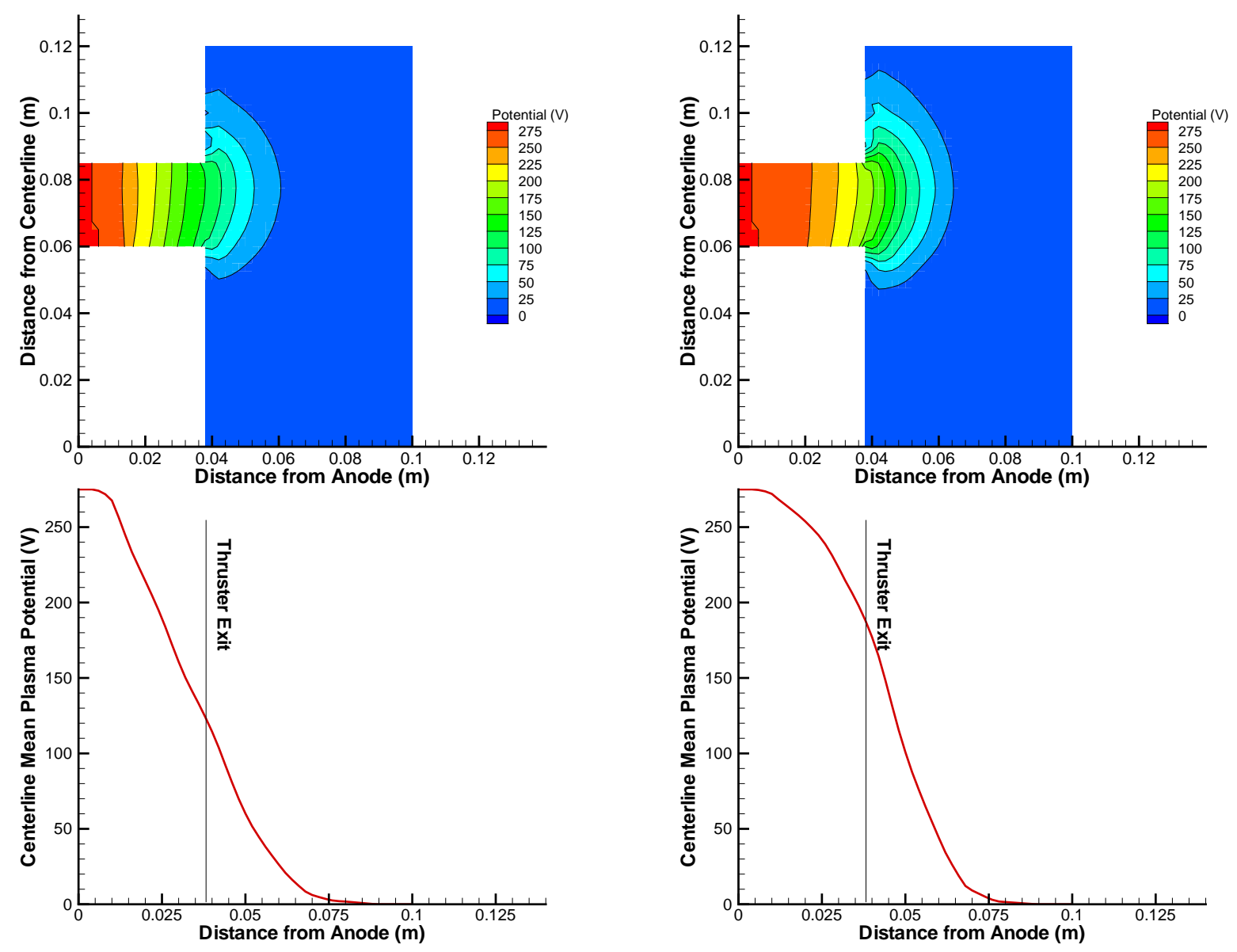

(a) Low Bohm

(b) High Bohm

Figure 3. UM/AFRL P5 Centerline Mean Potential Profiles (Part I) 

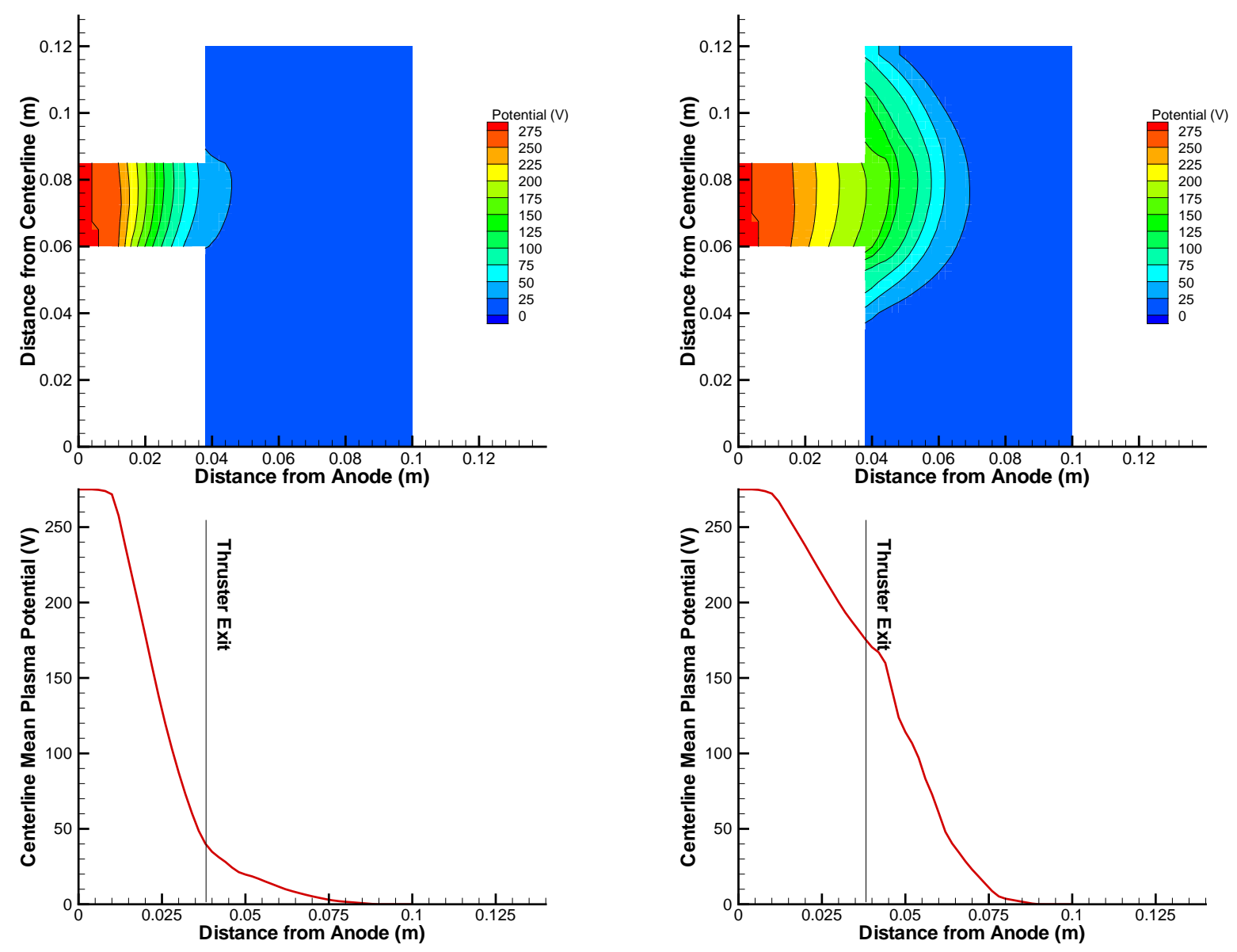

(a) Mixed Outer

(b) Mixed Inner

Figure 4. UM/AFRL P5 Centerline Mean Potential Profiles (Part II) 


\section{Acknowledgments}

The first author gratefully acknowledges financial support from the University of Michigan, the Department of Energy through a Computational Science Graduate Fellowship, and NASA Glenn Research Center.

\section{References}

${ }^{1}$ Koo, J. W. and Boyd, I. D., "Boundary Conditions for a 2-D Hybrid Stationary Plasma Thruster Model," AIAA-2004-3781, 40th AIAA Joint Propulsion Conference, Fort Lauderdale, FL, July 2004.

${ }^{2}$ Koo, J. W. and Boyd, I. D., "Computational Model of a Hall Thruster," Computer Physics Communications, Vol. 164, No. 1-3, December 2004, pp. 442-447.

${ }^{3}$ Fife, J. M., Hybrid-PIC Modeling and Electrostatic Probe Survey of Hall Thrusters, Ph.D. thesis, Massachusetts Institute of Technology, Department of Aeronautics and Astronautics, Cambridge, MA, 1998.

${ }^{4}$ Hagelaar, G. J. M., Bareilles, J., Garrigues, L., and Boeuf, J.-P., "Two-dimensional model of a stationary plasma thruster," Journal of Applied Physics, Vol. 91, No. 9, May 2002, pp. 5592-5598.

${ }^{5}$ Haas, J. M., Low-Perturbation Interrogation of the Internal and Near-Field Plasma Structure of a Hall Thruster Using a High-Speed Probe Positioning System, Ph.D. thesis, University of Michigan, 2001.

${ }^{6}$ Boeuf, J.-P. and Garrigues, L., "Low Frequency Oscillations In a Stationary Plasma Thruster," Journal of Applied Physics, Vol. 84, 1998, pp. 3541-3544.

${ }^{7}$ Morozov, A. I. and Shubin, A. P., "Electron kindetics in the wall-conductivity regime I and II," Sov. J. Plasma Phys., Vol. 10, No. 6, 1984, pp. 728-735.

${ }^{8}$ Esipchuck, Y. V. and Tilinin, G. N., "Drift Instability in a Hall-Current Plasma Accelerator," Sov. Phys. - Tech. Phys., Vol. 21, 1976, pp. 419. 